\title{
MMP-2 and MMP-9 in human peripheral blood: \\ optimizing gelatinase calibrator for degradome research and discovering a novel gelatinolytic enzyme
}

\section{Daniela Ligi ${ }^{1}$, Rosanna Maniscalco ${ }^{1}$, Ferdinando Mannello ${ }^{1 *}$}

${ }^{1}$ Department of Biomolecular Sciences, Section of Clinical Biochemistry and Molecular Genetics, University of Urbino Carlo Bo, Urbino, Italy.

*Corresponding author: Prof. Ferdinando Mannello, Department of Biomolecular Sciences, section of Clinical Biochemistry and Molecular Genetics, University of Urbino Carlo Bo, via O. Ubaldini, 7, 61029, Urbino, Italy. Phone: +39-0722-304621

E-mail: ferdinando.mannello@uniurb.it 


\section{SUPPORTING INFORMATION:}

Table S1. Gelatin zymography protocol for separating and stacking gel (100:1 Acrylamide:crosslinker).

Table S2. Composition of gelatinase calibrators obtained from peripheral blood.

Table S3. Size differences between $2.6 \% \mathrm{C}$ and $1.0 \% \mathrm{C}$ zymogram gels.

Table S4. Advantages and disadvantages of preparing calibrators according to STD $\mathrm{SDS}$ Vs. STDEIB method.

Table S5. Features of VHMW gelatinase arising from experimental observations.

Figure S1. Freeze-thawing stability of gelatinase calibrators STD SDS $_{\text {and }}$ STDEIB $_{\text {after } 1,4,10}$ freeze/thawing $(\mathrm{F} / \mathrm{T})$ cycles.

Figure S2. Gelatin zymography of STDs with and without APMA treatments and in presence of Caand Zn-chelators.

Figure S3. Gelatin zymography of $\mathrm{STD}_{\mathrm{EIB}}$, serum samples diluted in $\mathrm{EIB}$ or $\mathrm{NaCl}$, treated with and without APMA. 
Table S1. Gelatin zymography protocol for separating and stacking gel (100:1 Acrylamide:cross-linker).

$\mathbf{7 . 5 \%}$ Separating gel 4\% Stacking gel $+0.3 \%$ gelatin

\begin{tabular}{rcc} 
Distilled water & $3,850 \mu \mathrm{L}$ & $1,503 \mu \mathrm{L}$ \\
\hline 30\% acrylamide:PDA 100:1 (w/v) & $2,500 \mu \mathrm{L}$ & $333 \mu \mathrm{L}$ \\
$\mathbf{1 . 5}$ M Tris HCl pH 8.8 & $2,500 \mu \mathrm{L}$ & --- \\
0.5 M Tris HCl pH 6.8 & --- & $625 \mu \mathrm{L}$ \\
\hline 3\% gelatin 90 Bloom solution (w/v) & $1,000 \mu \mathrm{L}$ & --- \\
$\mathbf{1 0 \%}$ SDS solution & $100 \mu \mathrm{L}$ & $25 \mu \mathrm{L}$ \\
$\mathbf{1 0 \%}$ ammonium persulfate (w/v) & $50 \mu \mathrm{L}$ & $14 \mu \mathrm{L}$ \\
TEMED solution & $7.5 \mu \mathrm{L}$ & $7 \mu \mathrm{L}$ \\
Final volume & $10,0075 \mu \mathrm{L}$ & $2,507 \mu \mathrm{L}$
\end{tabular}


Table S2. Composition of gelatinase calibrators obtained from peripheral blood.

\begin{tabular}{|ccc|}
\hline STDSDS & STDEIB \\
\hline Peripheral blood & $18 \mu \mathrm{L}$ & $18 \mu \mathrm{L}$ \\
\hline Native sample buffer & $162 \mu \mathrm{L}$ & --- \\
\hline Native sample buffer $+\mathbf{8 . 4 \%}$ SDS & $90 \mu \mathrm{L}$ & $* 90 \mu \mathrm{L}$ \\
EIB & --- & $162 \mu \mathrm{L}$ \\
\hline Final volume & $270 \mu \mathrm{L}$ & $270 \mu \mathrm{L}$ \\
\hline
\end{tabular}

\footnotetext{
STD $_{\text {SDS }}$ was obtained by immediately adding $18 \mu \mathrm{L}$ of peripheral blood from the blood drop to a polypropylene tube containing $162 \mu \mathrm{L}$ of Native sample buffer and $90 \mu \mathrm{L}$ of Native sample buffer $+8.4 \%$ SDS, to obtain a final blood dilution

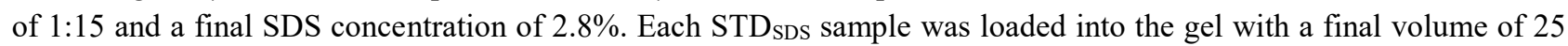
$\mu \mathrm{L}$, corresponding to a whole peripheral blood volume of $1.67 \mu \mathrm{L}$.

* STD $_{\text {EIB }}$ is obtained by immediately adding $18 \mu \mathrm{L}$ of peripheral blood from the blood drop to a polypropylene tube containing $162 \mu \mathrm{L}$ EIB (1:10 dilution). Native sample buffer $+8.4 \%$ SDS was added only before the sample loading into the well, in a proportional manner (i.e. $1 / 3$ of the final loading volume of $25 \mu \mathrm{L}$ ). This 2 -step STDEIB preparation allowed us to avoid the interference of SDS detergent contained into the native sample buffer+SDS for gelatinase extraction from

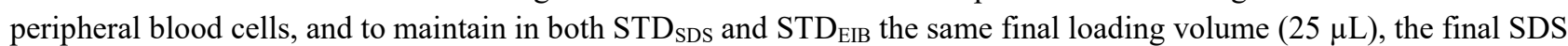
concentration $(2.8 \%)$ and the final whole peripheral blood loading volume $(1.67 \mu \mathrm{L})$.
} 
Table S3. Size differences between $2.6 \% \mathrm{C}$ and $1.0 \% \mathrm{C}$ zymogram gels.

\begin{tabular}{lcc}
\hline & $\begin{array}{c}\mathbf{2 . 6 \%} \\
\text { Length } \mathbf{x} \text { Height, cm } \\
\text { (thickness, mm) }\end{array}$ & $\begin{array}{c}\mathbf{1 . 0 \%} \\
\text { Length } \mathbf{~ H e i g h t , ~ c m ~} \\
\text { (thickness, mm) }\end{array}$ \\
\hline $\begin{array}{l}\text { Effective space between } \\
\text { glasses of mini-gel system }\end{array}$ & $8.2 \times 7.3$ & $8.2 \times 7.3$ \\
\hline $\begin{array}{l}\text { Mean gel size after } \\
\text { incubation }( \pm \mathrm{SD})\end{array}$ & $9.1 \pm 0.2 \times 7.8 \pm 0.2$ & $(1.5)$ \\
\hline
\end{tabular}

Measures were taken from zymogram gels casted with a 7.5\% fixed-concentration separating gel and a $\mathrm{T}=4 \%$ stacking gel $\left({ }^{*}=\mathrm{p}<0.05\right.$, calculated through one-way ANOVA for $2.6 \%$ vs. $1.0 \%$ mean gel size after incubation vs. effective space between glasses of mini-gel). 
Table S4. Advantages and disadvantages of preparing calibrators according to STD $\mathrm{SDS}$ vs. STDEIB method.

\begin{tabular}{|l|}
\hline \multicolumn{1}{|c|}{ STD } \\
\hline \multicolumn{1}{|c|}{ Advantages } \\
\hline Increased sample stability over time at RT \\
\hline Improved stability of gelatinolytic activity after multiple freeze-thawing cycles \\
\hline Compatibility with post-extraction treatments \\
\hline Simple and inexpensive method Disadvantages \\
\hline \multicolumn{1}{c|}{ STD } \\
\hline 2-step procedure \\
\hline \multicolumn{1}{c|}{ Advantages } \\
\hline Disadvantages \\
\hline Ready-to-load sample \\
\hline SDS-induced gelatinase activation \\
\hline Sample degradation over time at RT \\
\hline Incompatibility with post-extraction treatments \\
\hline Sample degradation during multiple freeze-thawing cycles \\
\hline
\end{tabular}


Table S5. Features of VHMW gelatinase arising from experimental observations.

\begin{tabular}{|c|}
\hline VHMW features \\
\hline $\mathrm{M}_{r}$ of 270-450 kDa \\
\hline APMA-activated form \\
\hline Covalently-linked multimer complex of MMP-9 \\
\hline Absence of a corresponding zymogenic form detectable in 4-8\% gradient zymography gel \\
\hline Observed in whole peripheral blood and weakly in serum only after APMA activation \\
\hline Inhibited by EDTA and 1,10-phenantroline \\
\hline Not observed in citrated plasma and WBC lysates \\
\hline Likely associated to cells participating in the coagulation process (platelets?) \\
\hline
\end{tabular}




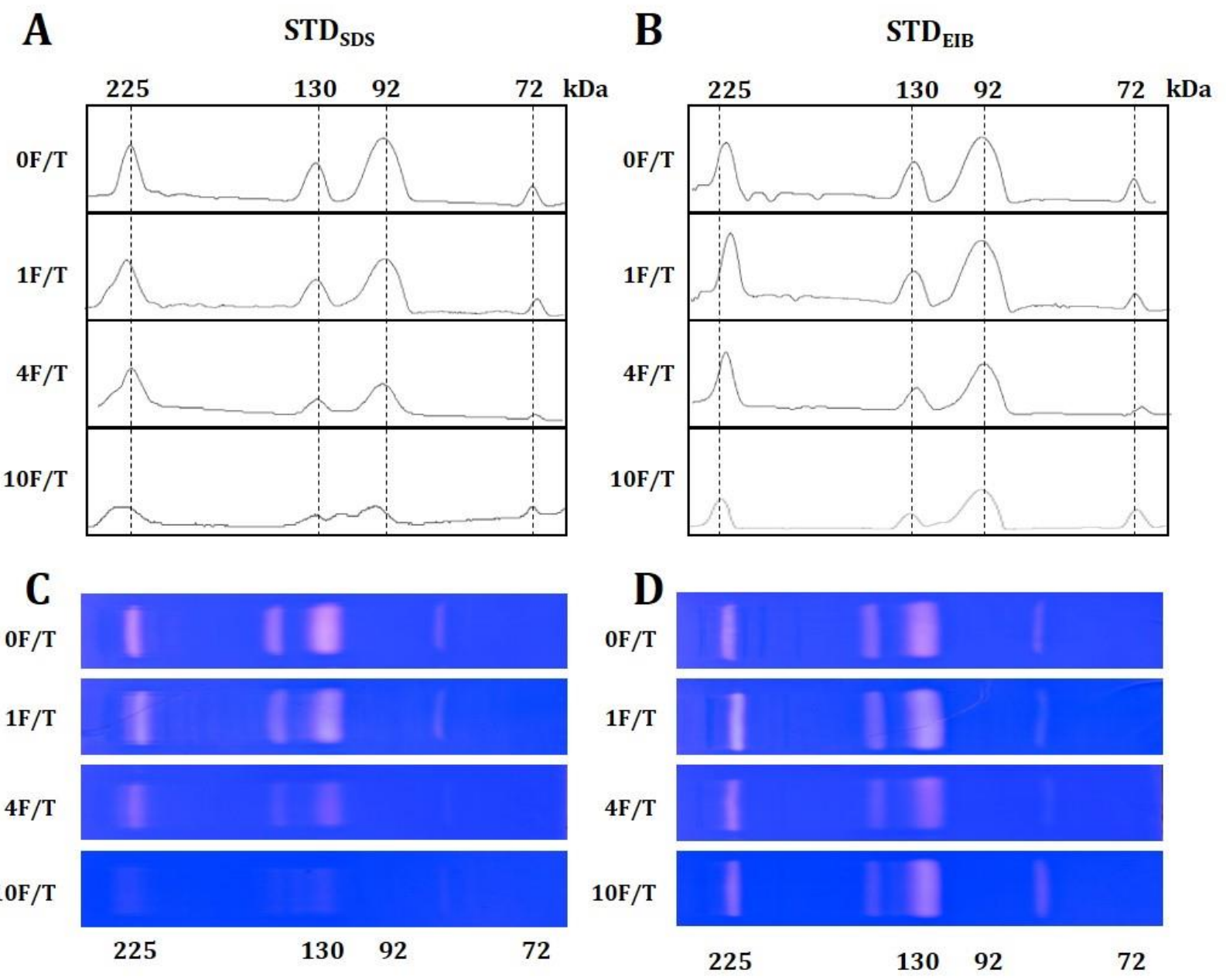

Figure S1. Freeze-thawing stability of gelatinase calibrators STD SDS $_{\text {and }}$ STD $_{\text {EIB }}$ after 1, 4, 10 freeze/thawing (F/T) cycles. Samples for freeze-thawing stability assays were overall stocked for a 4-months period. A, B) Schematic representation of gelatinolytic band measurement through densitometric analyses. Peaks of zymogenic gelatinases are reported as vertical dotted lines for $\mathrm{STD}_{\mathrm{SDS}}(\mathrm{A})$ and $\mathrm{STD}_{\mathrm{EIB}}$ (B). C, D) Representative gelatin zymographies (fixedconcentration separating gel of $7.5 \%$ and $1.0 \%$ of cross-linker) of STD $_{\mathrm{SDS}}(\mathrm{C})$ and $\mathrm{STD}_{\mathrm{EIB}}(\mathrm{D})$. 


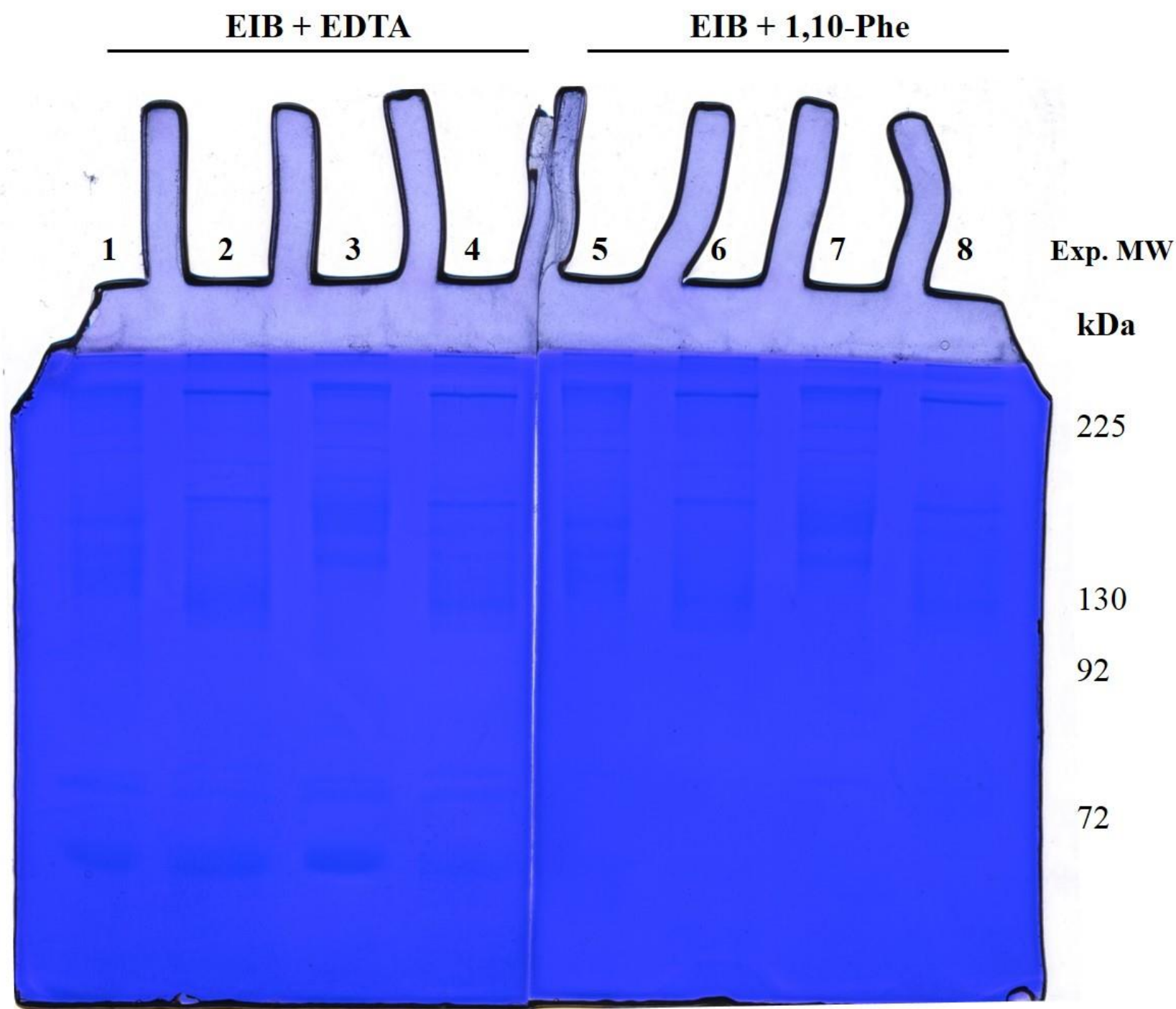

Figure S2. Gelatin zymography of STDs with and without APMA treatments and in presence of Ca- and Zn-chelators. Gelatin zymography was performed with fixed-concentration separating gel (7.5\%) and 1.0\% C. On the right are reported the expected molecular weights of zymogenic gelatinases. The left half-side of the gel has been incubated with EDTA 10 $\mathrm{mM}$; the right half-side of the gel has been incubated with 1,10-phenantroline $1 \mathrm{mM}$. Both gel sides have been incubated

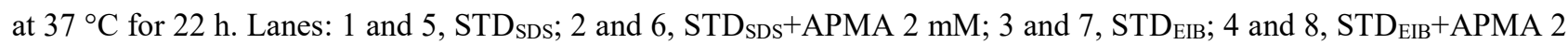
$\mathrm{mM}$ 


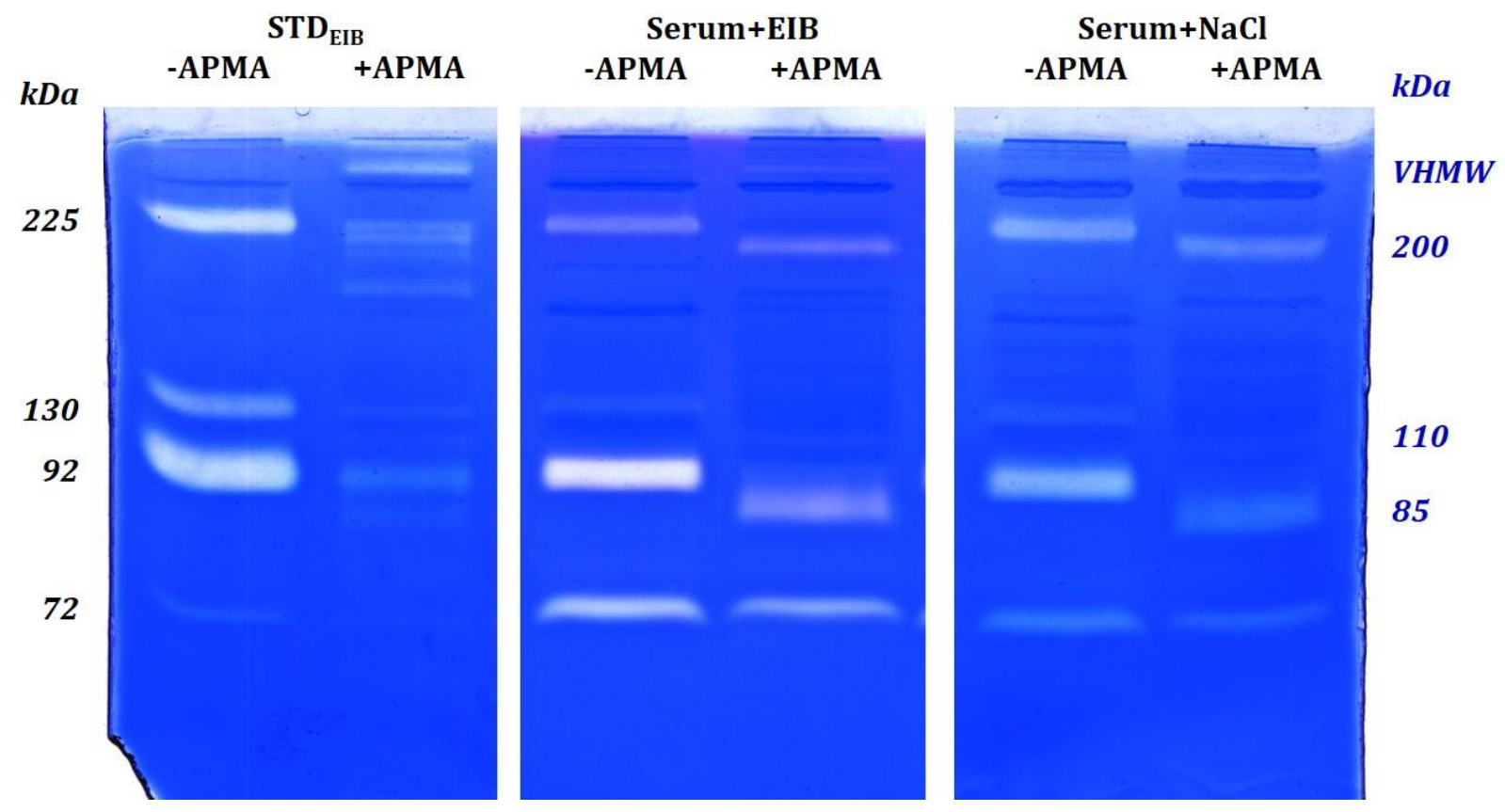

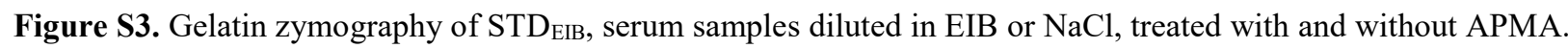
Gelatin zymographies were performed with fixed-concentration separating gel (7.5\%) and 1.0\% C. On the left are reported the reference molecular weight for zymogenic gelatinases; on the right side of the gel are reported the approximately calculated MW obtained from densitometric analyses of APMA activated forms. Standard of peripheral blood was extracted in EIB, as described in Materials \& Methods. Serum samples were diluted in EIB or in $\mathrm{NaCl} 0.9 \%$, and were incubated for $6 \mathrm{~h}$ at $37^{\circ} \mathrm{C}$ with or without $2 \mathrm{mM}$ of APMA. Serum were loaded to a final 350-375 $\mu \mathrm{g} / \mathrm{well}$ of total protein. (VHMW: Very High Molecular Weight). 